\title{
Resumen / Las artes mediáticas interactivas corroen el alma
}

El ensayo revela las diversas alternativas y problemáticas del arte en la actualidad, especialmente de las tendencias de fuerte impronta tecnológica, desde el video arte hasta lasmanifestacionesdigitales. Desde la perspectiva del análisis de la circulación y legitimación de obras y artistasen reconocidosfestivales, bienales y concursos internacionales, se reflexiona acerca del campo de las políticasculturalescomo espaciosen crisis frente a los grandes cambios del denominado espacio público. El texto plantea una mirada crítica de los factores económicos y políticos dominantes y su relación con lo tecnológico. Dentro de este contexto vincula la producción e inserción en el entramado cultural de las recientes manifestaciones del arte y delibera acerca del campo del arte y el rol en el contexto social de la gestión cultural y la curaduría.

\section{Palabras clave}

Arte - arte digital - arte experimental - audiovisual - cultura - curaduría de arte - gestión cultural mass media - política cultural - tecnología

\section{Summary / Interactive media arts wear aw ay the soul}

This essay reveals the different alternativesand problems of art nowadays, especially as regardsthose trends with a strong technological presence, from the video art to digital manifestations. From the perspective of the analysis of circulation and legitimating of worksand artists in well known festivals, biennial exhibitions and international contests, reflections ismade on the grounds of cultural policiesas areasin crisis on behalf of the big changes going on in the so called public space. The text proposes a critic view of the dominant economic and political factors and their relationship with technological issues. Within this context, it establishes a link between production and insertion of the most recent art manifestations into the cultural framework and discusses on the fields of art and the role in the social context of the cultural administration and curatorial work.

\section{Key words}

Art - art curatorial work - audiovisual - culture - cultural administration - cultural policies - digital art experimental art - mass media - technology

\section{Resumo / As artes mediáticas interativas corroem a alma}

O ensaio revela as diversasalternativase problemáticas da arte na atualidade, especialmente dastendências de forte estamparia tecnológica, desde o vídeo- arte até as manifestações digitais. Desde a perspectiva da análise da circulação e legitimação de obras e artistas em reconhecidos festivais, bienais e concursos internacionais, se reflexiona respeito do campo das políticas culturais como espaços em crise frente às grandes mudanças do denominado espaço público. O texto planteia uma mirada crítica dos fatores econômicos e políticos dominantes e sua relação com o tecnológico. Dentro desse contexto vincula a produção e inserção no tecido cultural das recentesmanifestações da arte e pondera respeito do campo da arte e o rol no contexto social da gestão cultural e a curadoria.

\section{Palabras chave}

Arte - arte digital - arte experimental - audiovisual - cultura - curadoria de arte - gestão cultural mass-média - política cultural - tecnologia

\footnotetext{
* Jorge La Ferla. Lic. Universidad de Paris VIII - Francia. Master In Arts - University of Pittsburgh - USA. Docente UBA, otras universidades nacionales e internacionales.

infocedyc@palermo.edu
} 
Considerando el ámbito en el que se plantean estas reflexiones, planteo el tema de la gestión cultural de eventos audiovisuales, como un lugar de crisis frente a los grandes cambios en la concepción del denominado espacio público. Las funciones de M icky Kwella son recordadas por la interesante combinación de animador cultural de eventos y activo curador, primero del VideoFest, y luego de su reconversión en la Trasmediale. Frente al entretenimiento uniforme que propone el sistema, a la pseudo globalización del mercado audiovisual, a la concentración mafiosofinanciera de la economía, y la predominancia del aparato digital en todos los procesos productivos es sugestivo revisar esta conflictiva situación.

Plantear esta temática implica relacionar estos trayectos de Kwella, con ciertas ideas de un pasado que pudieron predominar en el VideoFest, y otros organismos de su tipo alrededor del mundo en que el video fue en su momento el centro de la atención. Es interesante ver este proceso de escisión de las buenas viejas ideas y uto pías en este presente diverso. Un caso diverso en América Latina lo constituye actualmente la permanencia de VideoBrasil ${ }^{1} 0$ las actividades del Centro Cultural Banco Itaú ${ }^{2}$, que mantienen una línea bastante diversa a la de sus pares alemanes, o argentinos, por sólo establecer dos comparaciones funcionales.

Así fue como cada edición de la Transmediale se constituyó para algunos realizadores de América Latina en uno de los eventos anuales a tener en cuenta en el panorama del audiovisual independiente internacional. Esta manifestación, así como el Festival Internacional de Cine de Berlín, fueron fundados como foros y vidrieras de una búsqueda con el audiovisual independiente, experimental o autoral. Es una tradición que Alemania exhiba, en su remozada capital unificada y globalizada, un programa internacional de obras y autores de todo el mundo. Estas pantallas solían estar fuera del circuito de los medios masivos. Gracias a estos eventos el público berlinés, una excepción exigente en el chato paisaje mediático europeo y mundial, tenía acceso a un panorama de la producción audiovisual independiente internacional.

Recuerdo mi emoción cuando en 1977 asistí en la sala del Zoo, a una película cubana que se exhibía en Festival Internacional de Cine de Berlín. Pude ver lo que en el festival de la muerte de la dictadura argentina estaba prohibido ver en ese momento. Así fue como, también por primera vez, crucé el muro, y expectante veía "otra película" alrededor de la socialista Berlin Alexander Platz. Eran otros tiempos. Por un lado la socialdemocracia tutelada por los aliados enfrentaba a Erich Honecker, jefe todopoderoso de la República Democrática Alemana, a su vez con sus propias tutelas soviéticas. Todos convivían románticamente en una Berlín, plagada en sus bordes por No Man's Land de check points rusos, ingleses y americanos, de los que el joven cine alemán y las representaciones de las obras de Brecht trazaban un entusiasmado e ingenuo estado de cosas. Numerosos exilados chilenos perseguidos por el régimen pinochetista elegían tanto Berlín Este, como el Oeste, para exilarse. Nunca imaginaron que el primer secretario de Alemania del Este terminaría su existencia exilado en Chile y que hasta demolerían "su" lugar, "la casa de lámparas de Erich Honecker". Todos, en ambos lados, eran de izquierda, y no todos, deseaban la caída del muro. Dulces años 70. En la Argentina, Chile, Brasil, Bolivia, Colombia, Ecuador, Uruguay, Paraguay seguían las dictaduras militares; y en Alemania, Baader Meinhof y sus amigos, deliraban por otro estado de cosas mientras nosotros, latinoamericanos, éramos reivindicados como exóticos trofeos de la lucha revolucionaria exhibidos en la generosa tierra de asilo europea.

El primer día que llegué a Berlín y a la sede de la muestra, la Casa Internacional de las Culturas del Mundo, mientras esperaba entrevistarme con M icky Kwella y con Michel Thoss, caminé largamente seducido a través del bosque animado Autopoiesis la instalación de Kenneth Rinaldo ${ }^{3}$. La sugerente escultura robótica del artista americano formaba parte de la Transmediale 2002 que acababa de terminar. La Haus der Kulturen der Welt se había configurado en el espacio diverso, conflictivo y transversal, entre dos eventos por los cuales había pasado, y estaba, M icky Kwella. Los tentáculos de la estructura de Rinaldo -llena de sensores, ojos infrarrojos, hojas de parra, y microprocesadores- amagaban rozarme, mientras yo pensaba en la relación de la sofisticada escultura robótica con los materiales low tech que yo iba a mostrar durante esos días.

El tema del audiovisual independiente y las relaciones entre máquinas audiovisuales y la creación artística sigue siendo el tema clave, en un espacio y tiempo de conflicto, debido a los cambios en la materialidad de los soportes tecnológicos, y a los movimientos políticos y económicos en el mundo. La predominancia del aparato digital en todos los procesos productivos - bélicos, espaciales, mediáticos y medicinales- y la pseudo globalización de un mercado, audiovisual incluido, han generado una dramática homogeneización de ciertos parámetros en la producción y la curaduría artística. Muchos no quieren recordar las interesantes relaciones con la historia del cine, la TV, y el video experimental, conceptualizando un nuevo universo a escribir, a través del "Big Bang" de las llamadas nuevas tecnologías. Más allá de un soporte en vías de desaparición, como fue el electromagnético, la defensa nominativa del video arte implicó desde su momento de esplendor hasta hoy, una clara posición ideológica planteada frente al sistema del arte audiovisual, más allá del soporte utilizado.

Al hablar de video experimental seguimos nominando lo alternativo y autoral, a partir de la búsqueda experimental y una ruptura con las formas del espectáculo. Esa idea conceptualiza aquellos logros "admirables" en la creación artística con los medios audiovisuales que en algunos pocos casos de la historia de los medios se convierten en trascendente. La formula: medios + obra + arte + experimental + independiente sigue siendo una fórmula subversiva de importante valor en un mundo dominado por unas pocas corporaciones que se mueven fuera del 
ámbito, y de las leyes, de estados e instituciones públicas y corporaciones privadas, en absoluta decadencia. Esas entelequias poseen como único modelo para el espectáculo audiovisual el beneficio económico y la programación chatarra. A través de un flujo caracterizado por un paupérrimo flujo audiovisual liderado por la TV, el nuevo orgullo de un hemisferio norte liderado por dudosos estadistas, empresarios y actores, son las nuevas tecnologías. Podemos mencionar algunas pocas instituciones y festivales que pelean por una diversidad de calidad y compromiso con el audiovisual. Hablamos de casos aislados, y cada vez más minoritarios, especialmente en una Europa que viene de ser presidida "per il cavaliere Berlusconi", cuya coalición de gobierno en Italia se conforma con partidos de ultra derecha filo fascistas. Los lugares independientes están cada vez más amenazados por los cortes de presupuesto y por funcionarios -de instituciones privadas, mixtas 0 semiestatales- muy temerosos de perder su puesto, que establecen la necesidad banal de promover un discurso que propicie el lucro con los medios audiovisuales o que logran vender como novedad la actualización del discurso sobre los medios audiovisuales a partir del uso del soporte digital. Así se fueron ubicando bajo el eufemismo de las nuevas tecnologías interactivas, un vacío ideológico difícil de disimular.

En su momento surgieron instituciones en Europa que suscitaron gran interés, como fue el caso del Centre International de Création Video, en Montbéliard (C.I.C.V. $)^{4}$; Zentrum für Kunst und M edientechnologie $(Z K M)^{5}$, en Karlsruhe, que plantearon durante la década de los 90 una propuesta inédita de eventos y festivales, de centros de producción e investigación en las artes audio-visuales y con los medios electrónicos y digitales.

Ambas instituciones referentes actualmente no están pasando por su mejor momento.

Destaco estos centros pues marcaron en Europa un foro de praxis y diferencia ideológica frente a la uniformidad en los usos y consumos de los medios audiovisuales. El C.I.C.V., renombrado luego, simbólicamente, Centre de Recherche Pierre Schaeffer, tiene el mérito de haber producido o participado en la creación de obras trascendentes que marcaron la historia del video ${ }^{6}$.

Curiosamente en su reciente apertura al tema digital, ya no se puede decir lo mismo, en cuanto a establecer un parnaso de grandes obras. Más bien es lo contrario, un desierto total. De todas maneras el C.I.C.V. se destaca, durante los años 90, en la historia del video experimental. Algo, sin dudas, difícil de conseguir.

Un curioso testimonio de estos estados ambiguos alterados, entre lo videográfico experimental y el arte digital interactivo, lo explicita el premio obtenido por el video experimental Vacas de Gabriela Golder? en la última convocatoria en ZKM del oneroso Internationaler Medien Kunst Preis $2003^{\circ}$. Un video simple, breve, de costo ínfimo se lleva el premio otorgado por una institución dedicada con obsesión al audiovisual digital inmersivo e interactivo, a la ro- bótica, al bioarte y a un pensamiento sofisticado sobre el arte del silicio ${ }^{9}$. Paradójico premio de notable valor agregado, si consideramos que Woody Vasulka, padre y artista antológico del cine y el video experimental del siglo $X X$, fue uno de los jurados de este premio.

Estimo que el referido, y quiero creer bien intencionado, homenaje a Micky Kwella permite repensar la esencia, la existencia y el espíritu de algunas de las instituciones y eventos que han propiciado el audiovisual en sus diversas manifestaciones en Europa. Recordemos que la Unión Europea tiene actualmente una incoherente política de incentivar "las nuevas tecnologías", algo que en muchos casos se presenta separado de la esencia del videoarte clásico pero que mantiene la coherencia con sus desprolijos programas, Media I y Media II, para cine, video y TV.

Por otra parte la situación institucional y económica de Latinoamérica vuelve complicado el mantenimiento de cualquier centro, festival, acervo cultural o artístico audiovisual. Esto es aún más complejo cuando se trata de material independiente. La gran excepción sigue siendo Videobrasil, un ejemplo de supervivencia del que quizás sea actualmente el festival más importante de video y artes electrónicas del mundo. Videobrasil es una de las pocas manifestaciones dedicadas al audiovisual que logra continuidad en el tiempo en América Latina. Videobrasil se destaca por mantener su esencia conceptual, considerando el video como esencia de las artes electrónicas habiendo incorporado a lo largo de estos años, paulatina e inteligentemente, el tema de las artes digitales interactivas. También el proyecto de investigación, muestra itinerante y de publicación, Made in Brazil (Machado, 2003), del Centro Cultural Banco Itaú se sitúa en esta línea de mantener un balance inteligente en algo que en Brasil es fundamental, como es considerar la historia del video arte brasileño antecedente trascendente del arte digital. Made in Brazil es el resultado de una investigación comprensiva sobre la historia del video arte en Brasil. Durante mucho tiempo, el VideoFest -luego la Transmediale- así como el Foro de Cine Joven del Festival de Berlín, junto con otros festivales en el hemisferio Norte fueron un lugar de referencia en que la producción audiovisual independiente de América Latina aspiraba mostrarse. Los jóvenes realizadores de video, y cine, tuvieron en estos eventos un lugar privilegiado para mostrar sus obras producidas en la soledad, por la falta de apoyo y presupuesto, pero que se destacaban por una notable libertad creativa. Esa mezcla de energías generó en Europa una interesante curaduría de obras artísticas alternativas de América Latina.

El video experimental, encontraba en su esencia tecnológica el marco para fragmentar todos los parámetros genéricos, particularmente el del documental, y profundizaba la hibridez de combinaciones con la fotografía, el cine, las instalaciones y el aparato digital produciendo discursos cada vez más alejados de la uniformidad del lenguaje cinematográfico. El video demostraba una vez más en el campo de los 
medios que la singularidad nunca se llevó bien con la búsqueda de consenso (Bergala, 1985) fragmentando la sistemática repetición de fórmulas de los medios masivos, particularmente el cine y la TV. modelo de emoción del cine comercial caracterizado por eliminar cualquier marca autoral a partir de fórmulas y modelos seriales. Ese lugar de referencia y búsqueda de originalidad precisamente lo sigue ocupando el video experimental, por los procesos de trabajo, por su autonomía creativa e independencia formal e ideológica.

En los años 80 con la aparición en el mercado de las configuraciones digitales de manejo y control de los equipos de video empezó un proceso irreversible de innovación tecnológica que fue el paso previo a la uniformización digital de todos los soportes, casi siempre a partir de las mismas marcas y programas. Las máquinas electrónicas y digitales de manipulación y transferencia en los aparatos de montaje se impondrían también en el cine, primero a través del video, y luego con el computador. Pero fueron pocos los que en ese momento se imaginaron un futuro dominado por el procesamiento digital del audiovisual, algo que convulsionó la historia de los medios. Los realizadores de cine y video se encontraron de manera compulsiva frente a la transferencia obligada de los procesos de montaje, edición y postproducción centralizada en el computador, pero sin utilizar esta posibilidad como materia de ruptura e innovación. Sin embargo surgen algunas obras trascendentes resultado de la imaginación aplicada en ese proceso de hibridez entre el cine, el video y el procesamiento digital, las Historia(s) del Cine ${ }^{10}$ de Jean-Luc Godard y Escrito en el cuerpo ${ }^{11}$ de Peter Greenaway son dos ejemplos notables. Sin embargo los trabajos pioneros, más osados y trascendentes fueron producidos por autores situados fuera de la máquina fílmica, Art of Memory ${ }^{12}$ de Woody Vasulka, Planetópolis ${ }^{13}$ de Gianni Toti, los trabajos de Peter Greenaway para TV ${ }^{14}$ y toda la obra de David Larcher ${ }^{15}$ son buenos ejemplos, por sólo citar algunos.

El concepto del video experimental sigue vigente, pues reivindica los procesos de trabajo, la autonomía creativa, la independencia formal, y a veces, la ideológica, dentro de un sistema aburrido que ya no produce resquicios en su uniformidad audiovisual. El arte digital interactivo, por su parte, tiene aún un largo camino por recorrer, y una intensa deuda por saldar con aquel mundo utópico planteado por el video arte y el cine experimental en toda la historia de los medios audiovisuales.

Con la aparición en el mercado de las configuraciones digitales de manejo y control de los equipos de video empezaría un proceso irreversible de reciclaje tecnológico. Ese fue el paso previo a la uniformización digital de todos los soportes, a partir de las mismas marcas y programas. Las máquinas electrónicas y digitales de manipulación y transferencia en los aparatos de montaje se impondrían también en el cine, primero a través del video, y luego con el computador. Pero fueron pocos los que en ese momento se imaginaron un futuro dominado por el procesamiento digital del audiovisual, algo que convulsiona la historia de los medios. Los realizadores de cine y video se encontraron de manera compulsiva frente a la transferencia obligada de los procesos de montaje, edición y postproducción centralizada en el computador. Pero son pocos los que utilizarían esa posibilidad como materia de ruptura e innovación. Paulatinamente fue surgiendo un pensamiento inteligente que partiendo del computador, en su especificidad como aparato y en el procesamiento digital de información, fue definiendo a través de la escritura brillantes opciones de creación artística que, especulaciones mediante, renovarían el discurso audiovisual y la historia de los medios. Esa palabra profética, y altamente seductora, hasta ahora ha tenido un nivel más interesante que su concreción en obras admirables. En algunos casos partiendo ambas, obra y palabra, del mismo autor ${ }^{16}$.

Así se señaló todo un nuevo marco de posibilidades, considerando no solo la máquina digital, en su especificidad y diferencia, sino una nueva era para la cultura de los medios y el arte, interactivo y digital. Procesamiento, programación, interface, interconectividad, inmersión, bioarte, vidas artificiales eran algunos de los nuevos parámetros que pregonados por los nuevos pastores y gurúes en el campo de la investigación, la teoría y la crítica que hicieron carne en el pensamiento de los funcionarios, gestores culturales, directores de muestras y festivales.

El cine, en sus casi 110 años de historia, produjo solo un puñado de grandes pensadores, Gilles Deleuze, Sergei Eisenstein, André Bazin, Jean-Louis Comolli, según los gustos, en este caso muy francófilos. Pero resulta claro que tendremos que esperar aún bastante para ver la aparición de pensamientos ontológicos cruciales sobre este tema apasionante. Sin embargo, varios autores y artistas ya vienen desarrollando una tarea pionera, que sin duda es un punto de partida retórico para una praxis de la creación multimedia, y del arte mediático digital aletargada en su concreción material. El interés predominante que siguen esas tendencias es buscar, pensar y exhibir exclusivamente proyectos artísticos con la máquina digital considerando el buen uso de sus especificidades. La creación de los premio software art y a la interactividad de la Transmediale apuntaban en esta dirección.

Sin embargo, es importante no olvidar los usos corporativos, militares, comerciales financieros del soporte digital por parte de un sistema que vende la ilusión de democratizar su máquina financiera y de guerra a través de un discurso propagandístico centrado en las nuevas tecnologías. En una gira por los más destacados centros de investigación y experimentación audiovisuales de Alemania ${ }^{17}$ durante el año 2001, Nils Röller, profesor ligado a la Escuela de Artes y Medios de Colonia, y a ZKM, en una reunión informal con José Carlos M ariátegui y con el que suscribe, citaba un notable centro de investigaciones espaciales en las afueras de Colonia, donde él consideraba que era posible observar reales investigaciones artísticas de vanguardia con nuevas tecnologías. Curiosamente Röller al día siguiente realizaba una brillante presentación en el Archivo 
Flusser $^{18}$ de la Escuela de Artes y Medios de Colonia. La vigencia del pensamiento de Vilèm Flusser es clave para repasar algunas cuestiones sobre los medios que para muchos han quedado en una confusión total. Cantidades de encuentros y exposiciones en todo el mundo intentan mostrar las maravillas de los usos artísticos, narrativos y expresivos del soporte digital sin plantear un debate, cultural, científico y político profundo sobre los usos de la tecnología digital y las máquinas, en la historia de las guerras, la codicia y la intolerancia del ser humano. Este reciclaje ideológico se evidencia en la mayor parte de instituciones, congresos y actividades ligadas al arte digital. El estado alemán siempre mostró coherencia cuando a lo largo de la historia del siglo XX financia centros de producción de obras mediáticas y telemáticas ${ }^{19}$. Otro lugar sintomático puede ser el Centre for $A d$ vanced Studies, que funciona casualmente en el seno del M.I.T., otro lugar de referencia para muchos, que testimonia en sus espacios las trayectorias del poder de las tecnologías y de sus derivaciones militares y medicinales. En este centro en el que se han logrado notables modelos de la bomba Napalm, el cual consigue prestigio dándole un trabajo alimenticio consistente a Noam Chomsky y a Nicholas Negroponte, produce incesantemente obras e investigaciones artísticas y científicas con un discurso alternativo que no logra ocultar los aparatos ideológicos de un lobby financiero poderoso, y de un estado nacional en desaparición cohesionado por el discurso evangelista presidencial y sus ineficientes juegos de guerra contra el Islam. Podríamos, parafraseando y leyendo de una manera un tanto elemental al maestro Flusser, alegar que desde el punto de vista del bien puro, no existe más que una diferencia de grado entre la creación artística, agradable al usuario, de una obra interactiva y de una bala, una bomba o un misil: En ambos se esconde el demonio: "Desde le punto de vista del bien puro, no existe más que una diferencia entre de grado entre el diseño elegante, y agradable al usuario, de una silla y un misil: en ambos se esconde el demonio. Pues ambos son funcionales." (Flusser, 2002) No solamente ambos son funcionales sino se han fabricado en los mismos espacios e instituciones.

En nuestra pobre Argentina solo podemos mencionar como centros mediáticos, las recordadas instituciones del estado que aplicaban la picana eléctrica para hacer hablar a los prisioneros de la dictadura militar. Este aparato de tortura, productor de textos de delación y dolor, fue mediáticamente usado por los militares y policías argentinos. Por cierto ellos siempre creyeron en el concepto de $\mathrm{Mac}$ Luhan de considerar la electricidad como información en estado puro.

El contexto de este nuevo milenio tras la guerra de Irak II recargada muestra que el eufemismo de la globalización de los años 90 se transformó en un presente de miseria y violencia inéditos para muchos de nuestros países. Desde el hemisferio Norte hasta países como Argentina, el extremo trágico, en diferentes grados se diluye la figura del estadonación protector en sus funciones democráticas pri- mordiales de garantía de salud, justicia, educación y equidad económica y social para sus ciudadanos. Es en este panorama local y mundial que los medios masivos predominan en su venta de consenso político y espectáculo barato, valga la redundancia. El cine independiente de autor, el video experimental y el arte digital como medio e ideología de lo alternativo e independiente han dejado de ser moda. Tomemos como ejemplo la retrospectiva planteada para el $54^{\circ}$ Festival Internacional de Cine de Berlín de 2004, dedicada a Hollywood, bajo el título Dificultades en el país de las maravillas. Los sweaters andinos, los cigarros de canabis del aquel público entusiasta de los años 70 del Festival de Berlín dejaron lugar a los vestidos largos, a los smokings y al éxtasis. Un Win Wenders maquillado y cansado aplaudía en el 2003 la película de Wolfgang Becker, Good Bye, Lenin elegida como mejor film por la Academia del Cine Europeo.

Siguiendo esta moda parecería ser que el único parámetro a tener en cuenta para los próximos festivales de artes mediáticas electrónicas, será la propuesta interacción / interface para las obras artísticas centradas en el computador, como lo expresara públicamente una de las curadoras de la Transmediale en el primer encuentro Emoçao art.ficial ${ }^{20}$, lamentando lo poco interactivo de muchas obras electrónicas en América Latina. Estos parámetros no suelen considerar otros aspectos ideológicos y formales de la historia y realidad del audiovisual. Particularmente la búsqueda de obras artísticas trascendentes y rupturales.

Prueba de esto podría ser la interesante exposición Future Cinema, The Cinematic Imaginary After Film, realizada entre los años 2002 y 2003 en ZKM donde hubo varias obras que testimoniaron de este estado de cosas de las artes mediáticas, y de sus paradojas inmersivas. La obra más elocuente Illuminated Average \# 1 Hitchcock Psycho, de Jim Campbell, era una diapositiva proyectada, sin ninguna propuesta de interactividad. Este ícono que muestra el ADN de la obra de Hitchcock, resulta en una imagen condensada de todos los fotogramas de la película, y en su aparente simpleza era una de las propuestas conceptualmente más complejas de toda la exposición. La instalación interactiva inmersiva SI POTERIS NARRARE ${ }^{21}$, LICET, de Jean-M ichel Bruyère, quizás era lo más sofisticado, vanguardista y oneroso en el ámbito tecnológico de la exposición, obra que no llegaba a tener la mínima elocuencia narrativa, interés, ni energía artística si la comparamos con los elocuentes y simples videos del mismo autor y su troupe ${ }^{22}$. Así queda la idea de que efectivamente no pueden, o no saben, narrar y/o emocionar a través de la máquina digital.

Uno de los pocos trabajos alemanes presentes en la exposición, de hecho uno de los mejores, era The Invisible Shape of Things Past ${ }^{23}$, el cual pone en la escena digital posibles lecturas de la construcción del espacio y el tiempo, entre la arquitectura y el cine, donde lo virtual se convierte en el único lugar posible para concretizar una propuesta única para reconstruir una memoria urbana histórica audiovi- 
sual. Esta obra admirable, de las más destacadas de la muestra, tal vez necesitaba estar acompañada por otros trabajos producidos por la misma productora, ArtCom $^{24}$ de Berlín. Me estoy refiriendo a los encargos para la empresa Mercedes Benz que creo también responden y se relacionan con el tema de la exposición, la imaginería cinemática después del cine. Recordemos que esta marca, emblema y símbolo de Alemania, está ya diseñando para el mercado autos provistos de pantallas de cristal líquido multifunciones, con conexión por antena on line, GPS, parabrisas de cristal líquido.

Varios pensadores están intentando dilucidar el increíble evento mediático ocurrido alrededor del caso de canibalismo juzgado en la ciudad de Kassel, sede de Documenta, uno de los eventos artísticos más importantes de Europa. Este triste espectáculo alrededor del impactante suceso protagonizado por el técnico de computadoras Armin M eiw es y su acción de muerte con el ingeniero Bernd Juergen Brandes son el resultado de un sintomático modelo patológico de las sociedades desarrolladas. Peligroso aviso que pocos quieren asumir en todas sus implicancias. El hecho en sí mismo y los procesos mediáticos que lo circundan ponen en una preocupante escena este horroroso crimen, único en la historia europea, que llevan a un paroxismo preocupante cualquier elucubración sobre un llamado de atención sobre un estado de cosas que nadie quieren ver en todas sus dimensiones. Y por lo tanto, pocos pueden analizar, en sus razones, símbolos y consecuencias.

"Alemania va hacia delante" declaraba eufórico Gerhard Schröder a fines del año 2003 cuando el parlamento alemán aprueba los recortes de presupuestos destinados a desempleo y bienestar social, que explícitamente anuncian los próximos recortes en educación, arte y cultura.

"La política como continuación del arte con otros medios" (Günter,1989), decía como posible respuesta el artista Ingo Günther. Otros personajes, ligados a instituciones de fomento audiovisual que reciben financiamiento estatal, siguen la política del avestruz, temerosos de perder sus puestos y salarios. Ahora bien, dentro de este contexto me pregunto, ¿dónde están los herederos de Joseph Beuys, Wolf Vostell, Klaus vom Bruch y Michael Klier? ${ }^{25}$ Es decir aquellos místicos de la búsqueda artística, el inconformismo, la independencia del sistema y la contundencia creativa.

Recordemos la paradoja de que dos obras cumbres experimentales de los años 90, admirables por sus

\section{Notas}

www.videobrasil.org.br

http://www.itaucultural.com.br

www.cgrg.ohio-state.edu/ rinaldo/westcoast. Recordamos que Rinaldo participó junto a Lucia Santaela de la primera conferencia de la serie "Interactividades" organizada por Rejane Cantoni y Daniela Kutschat en el Centro Cultural Banco Itaú en San Pablo en septiembre del 2003. http://www. itaucultural.org.br/interatividades2003/ 4 ww.cicv.fr

${ }_{5}$ www.cicv.fr

Podríamos mencionar a Parabolic People, Sandra Kogut; Planetópolis y Tupac Amauta, Gianni Toti y la participación en la producción, reconstitución y reformulación de parte de la obra de Irit Basri,
}

ontológicas y rupturales lecturas sobre Alemania, Alemania 90 nueve cero y Berlín 10/90, fueron proyectos de dos autores extranjeros.

Frente a una cuestión de fondo como es la perdida de la singularidad en los medios, nos seguimos cuestionando, ¿Cómo plantear la defensa de la alteridad y originalidad que resulten en obras admirables con los medios audiovisuales? Este milenio será apasionante por los desafíos que plantea la completa digitalización del aparato audiovisual y la confusa amalgama que se genera al fagocitarse el ordenador los otros medios y máquinas tradicionales, dentro un marco de hibridación total de soportes.

Poco se podrá esperar del poder político y de las corporaciones multimedia. Tampoco les corresponde a ellos fomentar, producir y exhibir productos audiovisuales de calidad con una marca de autor, de carácter experimental, con fines y usos artísticos. Ellos solo pueden producir lucro y consenso. Esto obviamente es una cuestión política que nunca será modificada por los gobernantes de turno, frente a lo cual se nota una indefensión muy grande, por parte de funcionarios, gestores y administrado res culturales. En Argentina, demás está decirlo, el caso parece perdido. Creemos que el gran desafío es intentar mantener el espíritu conceptual, y radical, en la búsqueda y promoción de autores y artistas notables en el audiovisual, en todos sus soportes y combinaciones. Actitud particularmente peligrosa, por no decir subversiva, enfrentada al consenso del espectáculo y a la insoportable banalidad de ciertos conceptos alrededor de las nuevas tecnologías per se.

La defensa de esa alteridad es cada vez más difícil, y peligrosa, pero vale la pena mantenerla y sin temer hacer explícito el disenso.

Quizás varios factores atentaron contra la presencia de público en el último evento, medio@arte latino, organizado de manera casi solitaria por M icky Kwella en febrero de 2002 poco tiempo antes de su muerte. ${ }^{27}$

"- It was my biggest flop", me dijo Kwella, mientras fumaba y tomaba un café doble en la Haus der Kulturen der Welt.

Yo pensaba lo contrario. Más allá del dato aleatorio de la presencia masiva de público, el evento defendía un concepto y un criterio de curaduría. La buena organización, el bajo presupuesto y sus consignas mantenían una mística y una ideología, de la cual muchos se olvidaron, o definitivamente perdieron, en Alemania, en Europa y en el contexto mundial del audiovisual.

Será cuestión de recuperarlo.

Robert Cahen, Sandra Kogut, David Larcher, Marcello Mercado, Francisco Ruiz de Infante, entre otros. El CICV en 1991 auspició y produjo el Primer workshop de Video Creación a cargo de Eder Santos en Buenos Aires.

${ }_{7}$ Cows, Gabriela Golder, Argentina, 2002

www.medienkunstpreis.de o http:// swr.de/medienkunstpreis/ en/prizes.html

Me gusta el término definido por Santaela a arte do silício, Lucia Santaella. Publicado en la revista Cybercultura del Centro Cul tural del Banco Itaú, San Pablo, Brasil, 2003.

http://www. itaucultural.org.br/index.cfm?cd_pagina=2014\&cd_materia $=458$

${ }^{10}$ Histoire(s) du Cinéma, Jean-Luc Goōard, Francia, 1988/98 
${ }^{11}$ Pillow's Book, Peter Greenaway, Inglaterra, 1996

${ }^{12}$ Art of Memory de Woody Vasulka, USA, 1987

${ }^{13}$ Planetópolis de Gianni Toti, Francia/Italia, 1993

${ }^{14}$ TV Dante, Peter Greenaway y Tom Philipps, Inglaterra, 1988.

${ }^{15}$ Considero el más notable Video Void. The Trailer, David Larcher, Francia, 1993

${ }^{16}$ Los textos de Peter Weibel y Lev M anovich son notables en esta línea de pensamiento sobre el aparato digital. Cologne; German National Research Center for Information Technology (GMD), St. Augustin; Art\&Com, Berlin; Studio Babelsberg (digital studio center), entre otros.

Los participantes de este seminario fueron: Catarina Campino (Portugal), Stephanie Choo (Singapur), Sergio Edelsztein (Israel), Kathleen Forde (USA), Erdös Ibolya (Hungría), Kovylina Elena Kuenstlerin (Ucrania), Deborah Lawler-Dormer (Nueva Zelandia), Sonia Khurana (India), Piotr Krajewski (Polonia), Carlos Carlos Mariategui (Perú) , Gilbertto Prado (Brasil), Natasa Radosavljevic (Serbia), Karin Ohlenschläge (Alemania), Cleomar Rocha (Brasil), Margarita Schultz (Chile). Todos artistas, profesores y curadores, algunos con un nivel notablemente más alto que el de sus anfitriones teutones.

${ }_{19}^{18}$ Flusser-Archiv, Kunsthochshule für Medien Köln, www.khm.de ${ }^{19}$ Waypointing Weibel's Karlsruhe: Peter Weibel, director de ZKM durante la visita que organizamos junto al Goethe Institute de Peter

\section{Bibliografía}

-Bergala, Alain (1985) De la singularité, París: Cahiers du Cinéma. -Flusser, Vilèm (2002) La guerra y el estado de las cosas. (Der Krieg und der Stan der Dinge), Filosofía del Diseño. Madrid: Editorial Síntesis. -Frieling, Rudolf; Daniels, Dieter. (2002) Medien Kunst Interaktion. Viena: Goethe Institut, ZKM Zentrum für Kunst und Mediendtechno-
Weibel a Buenos Aires en el año 2002 comentaba que en el mismo espacio que funciona ZKM, en la ciudad de Karlsruhe, funcionó una fábrica de armas, más precisamente de balas y obuses, durante la segunda guerra mundial. Ver http://www.zkm.de/futurecinema/ weibel_werk_way_e.html

${ }^{20}$ Emoçao art.ficial, www.centroculturalbancoitau.org.br. San Pablo, Brasil, 2002.

Instalación Interactiva realizada y concebida por Jeffrey Shaw,

ZKKM, LFK-la fabriks, CICV-Pierre Shaeffer, 2002

Poèmes à linfect , 1997; De la vie des enfants au XXlème Siècle, Papisthione, 2000, La Fabriks/CICV.

The Invisible Shape of Things Past, Joachim Sauter http:// www.artcom.de/projects/invisible_shape/welcome.en

${ }_{24}^{24}$ Ver el Sector Negocios (Business) en www.artcom.de

${ }^{25}$ Hay pistas interesantes en la antología M edien Kunst Interaktion, notable trabajo de Rudolf Frieling Dieter Daniels, Goethe Institut, ZKM $1 / 2$ Zentrum für Kunst und M ediendtechnologie, Springer, Viena, 2002.

${ }^{26}$ Alemania 90 nueve cero, Jean-Luc Godard, 1991 ; Berlín 10/90, Robert Kramer, 1990 (Emisión Live, La Sept, Francia)

En ese mismo momento ocurría el Festival Internacional de Cine de Berlín, acababa de terminar la Transmediale, y además, hubo poda difusión mediática del evento.

logie, Springer.

-Günther, Ingo (1989) Kunstforum.

-Machado, Arlindo (org) (2003) Made in Brazil, Tres décadas de video brasileiro. SanPablo: Itaúcultural.

-Santaella, Lucía (2003) Revista Cybercultura, San Pablo: Centro Cultural del Banco Itaú. 